\title{
A PCR-based marker for targeting small rye segments in wheat background
}

\author{
M. Cristina Katto, Takashi R. Endo and Shuhei Nasuda* \\ Laboratory of Plant Genetics, Graduate School of Agriculture, Kyoto University \\ Sakyo-ku, Kyoto 606-8502 Japan
}

(Received 15 June 2004, accepted 30 July 2004)

\begin{abstract}
We attempted to develop a PCR-based marker that detects various segments of rye chromosome incorporated into wheat. We designed three sets of PCR primers based on the nucleotide sequence data of a rye repetitive sequence previously reported. One of the primer sets amplified a clear ca. $1.4 \mathrm{~kb}$ fragment in a rye cultivar but not in any form of wheat, diploid, tetraploid or hexaploid. We used this critical primer set for PCR of various wild species and cultivars of rye, an array of wheat plants carrying different rye chromosomes or small segments from different regions of rye chromosome $1 \mathrm{R}$, and plants carrying parts of the rye $\mathrm{B}$ chromosome. The PCR amplified the $1.4 \mathrm{~kb}$ fragment in all the plant materials examined. We believe this PCR primer set will be useful as a universal PCRbased marker for the introgression of rye chromosome segments in the wheat genome.
\end{abstract}

Key words: introgression, PCR, PCR-based marker, rye, wheat

Many wild and domestic Triticeae relatives of wheat (Triticum aestivum L.) are valuable sources of genes for wheat improvement (Graybosch 2001). These improvements include disease and pest resistance along with elevated protein content, and general agronomic traits. Rye (Secale cereale) is important as the major crop species in regions with adverse environmental conditions and is a source of alien chromosome segment containing agronomically useful genes for wheat breeding. Also, rye is a parent of the synthetic cereal Triticale (Philipp et al. 1994). There are many wheat cultivars carrying rye chromosome 1R, substituting for wheat chromosome 1B. The short arm of chromosome 1R (1RS) carries a number of genes of agronomic importance, and its introgression into wheat in the form of translocations with the long arms of wheat chromosome 1B (1RS.1BL) and 1A (1RS.1AL) resulted in some valuable new cultivars (Singh et al. 1990). The substitution of $1 \mathrm{R}$ for $1 \mathrm{~B}$ is considered to occur spontaneously. The origin of the rye source for German, Japanese and American 1RS.1BL translocations is different from each other (Schlegel and Korzun 1997). Besides chromosomes $1 \mathrm{R}$ and $1 \mathrm{RS}$, many other rye chromosome and chromosome arms have been introduced into wheat (Friebe et al. 1990, Friebe et al. 1994, Ribeiro-Carvalho et al. 2001, Lukaszewski et al. 2004). There have been attempts to transfer small rye segments into wheat by different means (Jiang et al.

Edited by Minoru Murata

* Corresponding author. E-mail: nasushu@kais.kyoto-u.ac.jp
1994). The most successful one so far achieved is the translocation between $1 \mathrm{R}$ and $1 \mathrm{~B}$ induced by homoeologous pairing (Lukaszewski 2000). Furthermore, a unique means using a gametocidal chromosome has been demonstrated to be effective in inducing translocations between $1 \mathrm{R}$ and wheat (Endo et al. 1998, Masoudi-Nejad et al. 2002).

Since smaller rye chromosomal segments are being introduced into wheat and larger populations need to be screened, a more reliable and easier means of detecting the alien segments, other than the chromosome banding and GISH techniques, is in demand. McIntyre et al. (1990) first reported the molecular marker (pSc119.1) specific to and ubiquitous on rye chromosomes. Specific PCR markers were designed to amplify chromosome 2R added to wheat (Lee et al.1993, Lee et al. 1995) or used to construct a linkage map in rye (Ma et al. 2001). A new class of transposon-like element designated Revolver was discovered to be distributed over the entire rye genome, but not at all in the wheat genomes (Tomita et al. 2003, Tomita and Yoshikawa 2003). Ko et al. (2002) showed by fluorescence in situ hybridization (FISH) that a random amplified polymorphic DNA (RAPD) product is present throughout the rye genome except at telomeric and nucleolar organizing regions. These studies suggested the presence of certain DNA sequences that are specific to rye but not to wheat. The PCR-based identification of such rye-specific sequences would be a useful way of detecting chromosomes and chromosomal segments of rye incorporated into the wheat genomes, with- 
out relying on the time-consuming procedures, such as Southern hybridization and in situ hybridization.

We cytologically analyzed the presence of rye chromosome segments in the plant materials shown in Table 1. We used one species of wild rye, seven cultivars of cultivated rye, ten rye-chromosome addition and two ryechromosome substitution lines of common wheat, three lines and a selection of 13 plants of common wheat carrying segments of rye chromosome $1 \mathrm{R}$, and a selection of three plants carrying parts of the rye $\mathrm{B}$ chromosome. We cytologically selected the two selections from the progeny containing the $1 \mathrm{R}$ and $\mathrm{B}$ segments that had been induced by the gametocidal system (Endo 2003). Three of the plants (122-5, 136-3 and 286-6) were homozygous and the remaining plants were hemizygous. Molecular cytological analyses were conducted as described by MasoudiNejad et al. (2002). Rye total genomic DNA labeled with digoxigenin-11-dUTP (Roche Applied Science), and a repetitive sequence specific to the rye $\mathrm{B}$ chromosome, D1100 (Sandery et al. 1990) labeled with biotin-11-dUTP (Roche Applied Science) were used as probes. A clone carrying D1100 was a kind gift from Neil Jones (University of Wales-Aberystwyth, Wales, UK).

Fig. 1 shows the images of genomic in situ hybridization of the small rye chromosome segments examined in

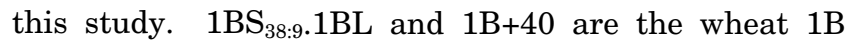
chromosomes with the smallest insertional and terminal translocations of the rye $1 \mathrm{R}$ satellite segments, respectively; the former has an interstitial segment (estimated at $1.7 \mathrm{cM}$ long) and the latter the telomeric C-band (Lukaszewski 2000). Lukaszewski (2000) identified the two translocation chromosomes by C-banding and by the presence or absence of the loci of seed storage proteins and disease resistance, whereas we clearly visualized the presence of rye segment in those chromosomes by GISH in this study. Line 1R-89 has the smallest $1 \mathrm{R}$ satellite terminal segments among the five wheat chromosome- $1 \mathrm{R}$ translocations studied by Masoudi-Nejad et al. (2002). The midget chromosome, or micro-chromosome, has one small proximal chromosomal fragment that was probably derived from chromosome $1 \mathrm{R}$ and had preferentially been maintained in a wheat line carrying the cytoplasm of rye (Murata et al. 1992).

The selection of 13 plants had small $1 \mathrm{R}$ segments from various regions of $1 \mathrm{R}$. The rye segments of 122-5, 126-3, 128-7 and 136-1 had deletions in both arms of $1 \mathrm{R}$ and therefore were from its proximal region. The $1 \mathrm{R}$ segments of 115-3, 117-10, 120-6, 126-6, 130-3, 133-4 and 136-3 were translocated onto and those of $112-1$ and 1165 were inserted into wheat chromosomes. The $1 \mathrm{R}$ segments of 112-1 and 126-6 probably came from the satellite because they had been coexisted with $1 \mathrm{R}$ chromosomes lacking terminal segments of the satellite in the parental plants. The $1 \mathrm{R}$ segments of $115-3$ and $120-6$ should be the terminal segments of the $1 \mathrm{R}$ long arm because they showed stronger GISH signals at the telomere and because they had been coexisted with $1 \mathrm{R}$ chromosomes lacking the distal regions of the long arm in the parental plants. The rye segments in the other plants arose from unknown chromosomal regions of $1 \mathrm{R}$. The B chromosomes of 281-2 and 286-6 had terminal deletions in the long arm, and 285-8 had an isochromosome of the long arm of the B chromosome.

Total DNA was extracted from all plants shown in Table 1 with Plant DNAzol reagent (Invitrogen). PCR primers were designed according to the nucleotide sequence of a rye-specific RAPD clone pSc20H (Ko et al. 2002, GenBank Accession Number AF305943). Three each of forward (F1 to F3) and reverse (R1 to R3) primers were designed near the 5 ' and 3' ends of the repetitive sequence; F1 (5'-GGGAG ACATC ATGAA CATTT-3'), F2 (5'-CATGA ACATT TGCAA AGTTG GA-3'), F3 (5'GATCG CCTCT TTTGC CAAGA-3'), R1 (5'-GGGAG ACATC TGTTG TTGGG-3'), R2 (5'-TGGGC AGAAA GGTCG ACATC-3'), and R3 (5'-TCACT GATCA CAAGA GCTTG-3'). These primers were expected to be rye-specific because Blast search did not reveal any identical sequences in the GenBank database, except for the pSc20H. PCR reaction was carried out in a $20 \mu \mathrm{l}$ mixture containing template DNA (ca. $100 \mathrm{ng}$ ), dNTPs (0.25 $\mathrm{mM}$ each), forward and reverse primers (20 pmol), $1 \mathrm{X}$ PCR buffer with $\mathrm{MgCl}_{2}(0.15 \mathrm{mM})$, and 2.5 unit of Taq polymerase (Roche Applied Science). The thermal cycling was programmed as follows: initial denaturation at $94^{\circ} \mathrm{C}$ for 5 min followed by 30 cycles of denaturing $\left(94^{\circ} \mathrm{C}, 30 \mathrm{sec}\right)$, annealing $\left(55^{\circ} \mathrm{C}, 30 \mathrm{sec}\right)$, and extension $\left(72^{\circ} \mathrm{C}, 90 \mathrm{sec}\right)$. All nine combinations of forward and reverse primers were tested with Petkus rye and Chinese Spring wheat. Only the primer combination F3 and R3 gave clear-cut results, i.e., amplification of a single-band PCR product (ca. $1.4 \mathrm{~kb}$ ) in rye but no amplification in wheat (Fig. 2). Other combinations of primers resulted in amplification in both rye and wheat, probably due to the presence of homologous sequence in the wheat genome. We used the critical primer combination for further study with various species or cultivars of rye, and the wheat lines and selections carrying parts of the rye genome.

PCR with the critical primer combination amplified the $1.4 \mathrm{~kb}$ band in all wild and cultivated rye species examined (Fig. 2). The primer set amplified no DNA in the hexaploid, tetraploid and diploid wheats used as controls. PCR with the same primer set amplified DNA of the same size in all rye chromosome addition and substitution lines tested, indicating that the critical DNA sequence is present in all seven chromosomes of Imperial rye, three chromosomes of the inbred rye, and two chromosomes of a wild rye, Secale montanum (Fig. 2). These results suggested that the critical DNA sequence is ubiquitous in the genus Secale. Furthermore, the PCR 
Table 1. Plant materials.

\begin{tabular}{|c|c|c|}
\hline & Rye chromosome segment & Source* \\
\hline \multicolumn{3}{|l|}{ Species, cultivar } \\
\hline Secale cereale cv. Imperial & Whole complement & NBRP \\
\hline Secale cereale cv. Prolific & Whole complement & NBRP \\
\hline Secale cereale cv. Petkus & Whole complement & NBRP \\
\hline Secale cereale cv. R16 & Whole complement & NBRP \\
\hline Secale cereale cv. R4 Podolia & Whole complement & NBRP \\
\hline Secale cereale cv. R81 & Whole complement & NBRP \\
\hline Secale cereale cv. Russian rye & Whole complement & NBRP \\
\hline Secale fragile & Whole complement & NBRP \\
\hline \multicolumn{3}{|l|}{$\underline{\text { Line }}$} \\
\hline$\overline{1 R^{\text {imp }}}$ addition & Chromosome $1 \mathrm{R}$ of Imperial rye & Lukaszewski \\
\hline $2 \mathrm{R}^{\mathrm{imp}}$ addition & Chromosome $2 \mathrm{R}$ of Imperial rye & Lukaszewski \\
\hline $3 \mathrm{R}^{\mathrm{imp}}$ addition & Chromosome 3R of Imperial rye & Lukaszewski \\
\hline $4 \mathrm{R}^{\mathrm{imp}}$ addition & Chromosome $4 \mathrm{R}$ of Imperial rye & Lukaszewski \\
\hline $5 \mathrm{R}^{\mathrm{imp}}$ addition & Chromosome 5R of Imperial rye & Lukaszewski \\
\hline $6 \mathrm{R}^{\mathrm{imp}}$ addition & Chromosome $6 \mathrm{R}$ of Imperial rye & Lukaszewski \\
\hline $7 \mathrm{R}^{\mathrm{imp}}$ addition & Chromosome 7R of Imperial rye & Lukaszewski \\
\hline $1 \mathrm{R}^{\mathrm{IR} 130}$ addition & Chromosome $1 \mathrm{R}$ of IR 130 & NBRP \\
\hline $2 \mathrm{R}^{\mathrm{IR} 130}$ addition & Chromosome $2 \mathrm{R}$ of IR 130 & NBRP \\
\hline $5 \mathrm{R}^{\mathrm{IR} 130}$ addition & Chromosome 5R of IR 130 & NBRP \\
\hline $1 \mathrm{R}^{\mathrm{m}}$ substitution & Chromosome $1 \mathrm{R}$ of $S$. montanum & PBI \\
\hline $2 \mathrm{R}^{\mathrm{m}}$ substitution & Chromosome $2 \mathrm{R}$ of $S$. montanum & PBI \\
\hline $1 \mathrm{BS}_{38: 9} \cdot 1 \mathrm{BL}$ & Translocation of $1 \mathrm{R}$ with wheat chromosome $1 \mathrm{~B}$ & Lukaszewski \\
\hline $1 B+40$ & Translocation of $1 \mathrm{R}$ with wheat chromosome $1 \mathrm{~B}$ & Lukaszewski \\
\hline 1R-89 & Translocation of $1 \mathrm{R}$ with wheat chromosome $7 \mathrm{D}$ & NBRP \\
\hline Midget & Micro-chromosome of wheat chromosome $1 \mathrm{~B}$ & Murata \\
\hline \multicolumn{3}{|l|}{ Selection } \\
\hline $112-1$ & Translocation of $1 \mathrm{R}$ with a wheat chromosome & This study \\
\hline $115-3$ & Translocation of $1 \mathrm{R}$ with a wheat chromosome & This study \\
\hline $116-5$ & Translocation of $1 \mathrm{R}$ with a wheat chromosome & This study \\
\hline $117-10$ & Translocation of $1 \mathrm{R}$ with a wheat chromosome & This study \\
\hline $120-6$ & Translocation of $1 \mathrm{R}$ with a wheat chromosome & This study \\
\hline $122-5$ & Deletion in both arms of $1 \mathrm{R}$ & This study \\
\hline $126-3$ & Deletion in both arms of $1 R$ & This study \\
\hline $126-6$ & Translocation of $1 \mathrm{R}$ with a wheat chromosome & This study \\
\hline $128-7$ & Deletion in both arms of $1 \mathrm{R}$ & This study \\
\hline $130-3$ & Translocation of $1 \mathrm{R}$ with a wheat chromosome & This study \\
\hline $133-4$ & Translocation of $1 \mathrm{R}$ with a wheat chromosome & This study \\
\hline $136-1$ & Deletion in both arms of $1 \mathrm{R}$ & This study \\
\hline $136-3$ & Translocation of $1 \mathrm{R}$ with a wheat chromosome & This study \\
\hline $281-2$ & Deletion in the long arm of B chromosome & This study \\
\hline $285-8$ & Isochromosome of the long arm of B chromosome & This study \\
\hline $286-6$ & Deletion in the long arm of B chromosome & This study \\
\hline \multicolumn{3}{|l|}{ Control } \\
\hline 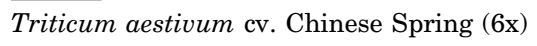 & None & NBRP \\
\hline Triticum aestivum $\mathrm{cv}$. Norin $61(6 \mathrm{x})$ & None & NBRP \\
\hline Triticum aestivum cv. Norin $26(6 \mathrm{x})$ & None & NBRP \\
\hline Triticum aestivum cv. Selkirk $(6 \mathrm{x})$ & None & NBRP \\
\hline Triticum aestivum $\mathrm{cv}$. Thatcher $(6 \mathrm{x})$ & None & NBRP \\
\hline Triticum paleocolchicum $(4 \mathrm{x})$ & None & NBRP \\
\hline Triticum carthlicum $(4 \mathrm{x})$ & None & NBRP \\
\hline Triticum monococcum $(2 \mathrm{x})$ & None & NBRP \\
\hline
\end{tabular}

*: NBRP: the National Bioresource Project-WHEAT, Japan (http://www.shigen.nig.ac.jp/wheat/komugi/). PBI: Plant Breeding Institute, UK.

Lukaszewski: A. Lukaszewski, Dep. of Botany and Plant Sciences, Univ. of California, Riverside, USA.

Murata: M. Murata, Research Institute for Bioresources, Okayama University, Japan. 

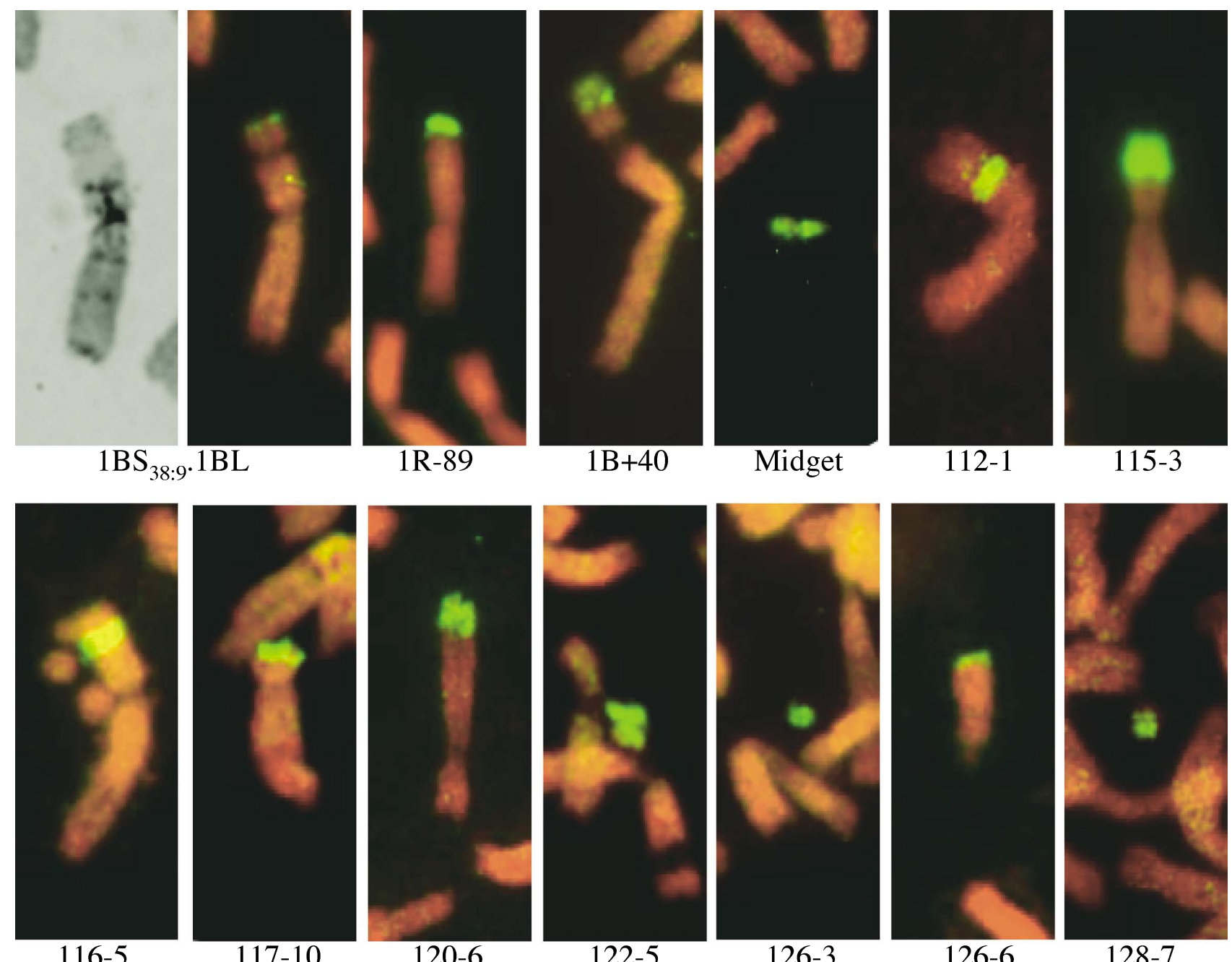

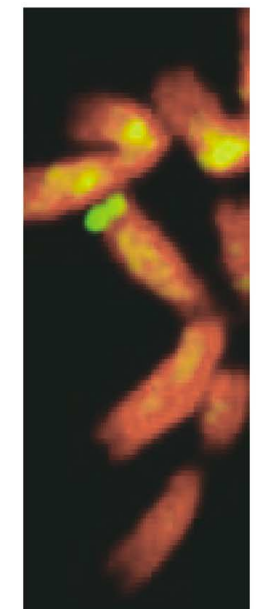

130-3

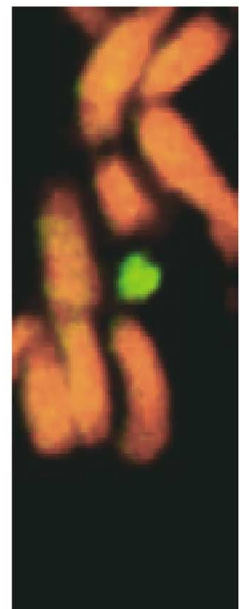

133-4

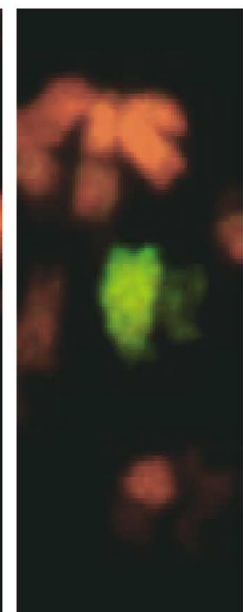

136-1

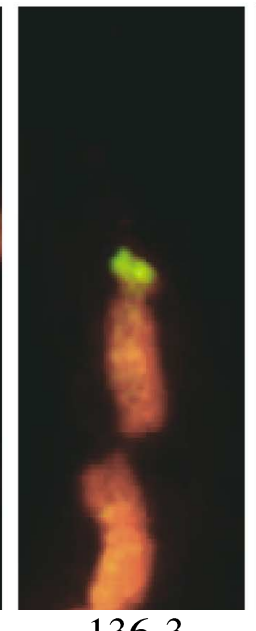

136-3

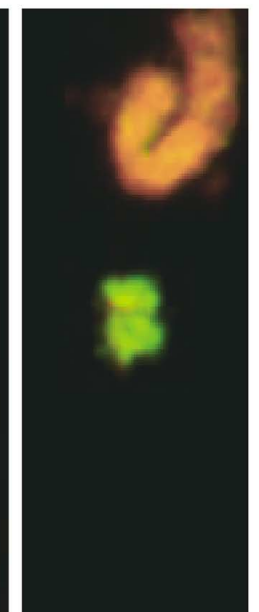

281-2

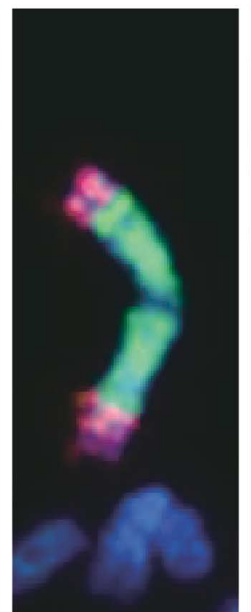

$285-8$

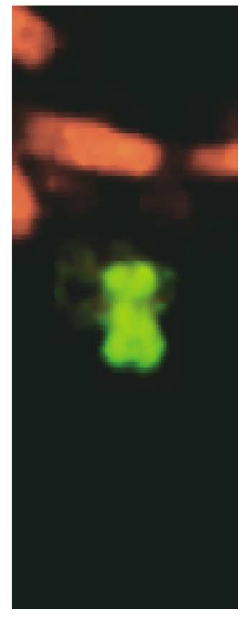

286-6

$10 \mu \mathrm{m}$

Fig. 1. Rye segments in the lines and selections analyzed in this study. The rye segments are shown in green fluorescein signals of GISH, with wheat chromosomes counterstained with propidium iodide in red (except for 285-8) or with 4',6-diamidino-2 phenylindole (285-8). The presence of a rye chromosome segment inserted into the $1 \mathrm{~B}$ satellite of $1 \mathrm{BS}_{38: 9} .1 \mathrm{BL}$ was demonstrated by the sequential C-banding (left) and GISH. The terminal B chromosome-specific repetitive sequence in 285-8 was detected by FISH, as shown in red signal. All photographs are in the same magnification and bar represents $10 \mu \mathrm{m}$. 


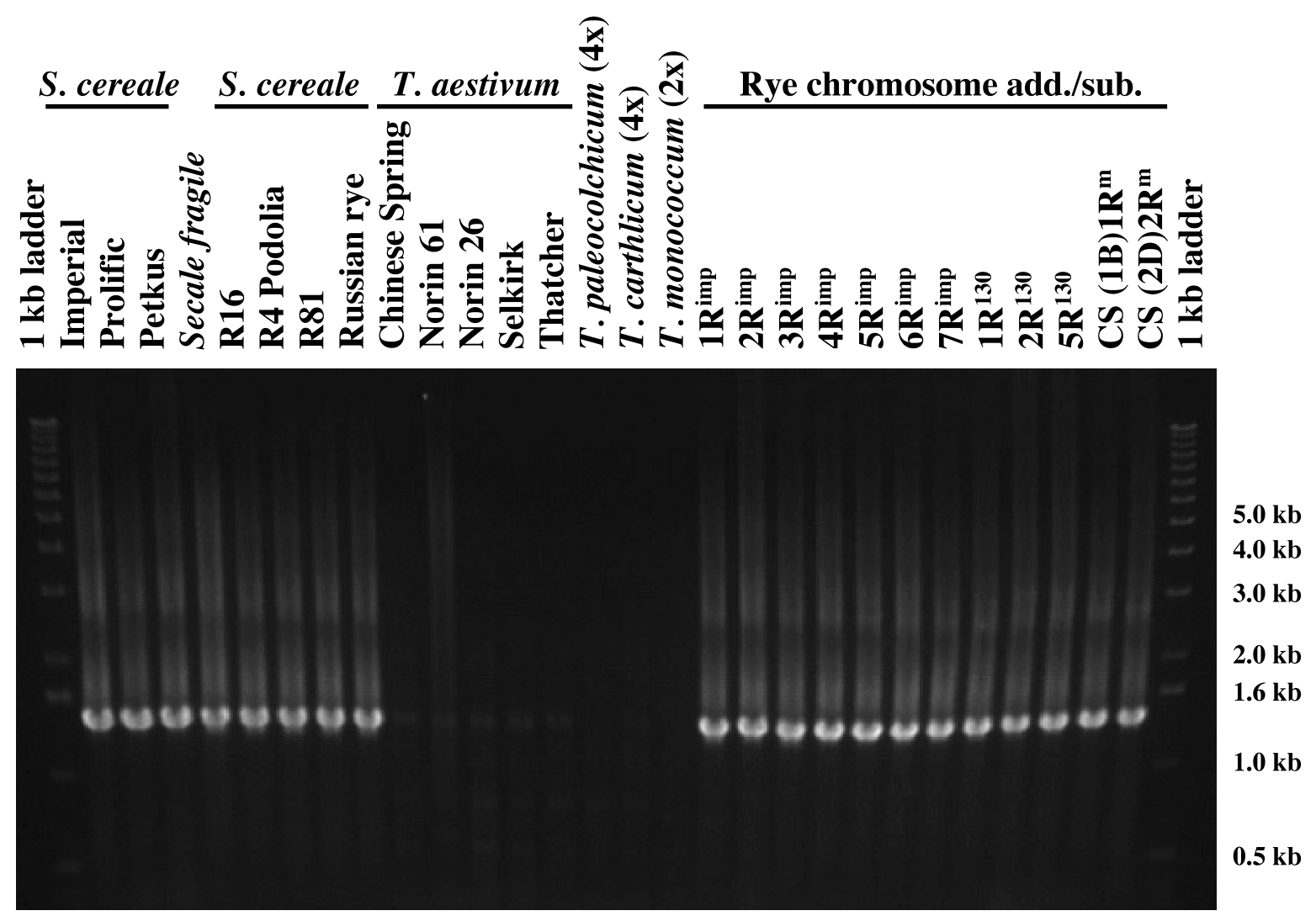

Fig. 2. An example of PCR assay for the presence of rye chromosome segments using the critical primer combination F3 and R3. Sample names are shown on the top of the panel. See Table 1 for detailed description of the lines tested. The fragment sizes of the molecular standard (1 kb ladder) are given on the left side of the panel. Note that ca. $1.4 \mathrm{~kb}$ PCR product was amplified in all plant materials containing rye segments from different sources, but there was no specific amplification in wheat.

amplification was observed in all lines and selections carrying rye segments (data not shown). This suggests that the critical DNA sequence is distributed widely in the $1 \mathrm{R}$ and $\mathrm{B}$ chromosomes.

Thus, this PCR marker detected various types of rye chromosome segment tested in this study, regardless of species, cultivars, chromosomes, and chromosomal segments. This broad applicability of the marker is due to the nucleotide sequence of the $\mathrm{pSc} 20 \mathrm{H}$, the original sequence of a rye specific RAPD marker (Ko et al. 2002). The sequence is homologous to part of the Ty3/gypsy-type retrotransposon. Although its copy number in rye genome is not clear, the FISH image probed with the $\mathrm{pSc} 20 \mathrm{H}$ clone indicated that the homologous sequence is abundant and ubiquitous in the rye chromosomes except for the telomeric regions (Ko et al. 2002). Ty3/gypsy retrotransposons are also abundant in the wheat genome. When the sequences homologous to the clone $\mathrm{pSc} 20 \mathrm{H}$ were searched in the DDBJ/GenBank/EMBL database, four wheat sequences (Accession numbers AY494981, AY188331, AY485644, and AF326781) were found in the top ten homologues. Sequence comparisons indicated that our successful rye-specific amplification was ensured by sequence diversity near the 3' termini of the F3 and R3 primers.

Generally, wheat lines carrying already-known rye chromosomes or chromosome arms are used to transfer small rye segments and genes into wheat. In practice, therefore, we do not need the chromosome-specific PCRbased markers developed to identify specific wheat-rye translocations, such as 1BL.1RS (Iqbal and Rayburn 1995; Koebner 1995), 2BL.2RS and 2BS.2RL (Brunell et al. 1999), and wheat-Imperial rye disomic addition lines (Gallego et al. 1998). Our PCR-based marker detected very small rye chromosome segments, including the one in $1 \mathrm{BS}_{38: 9} .1 \mathrm{BL}$, which would be easily overlooked by GISH (Fig. 1). PCR is a less technically demanding and more rapid, compared with the other techniques of chromosome mapping. The PCR primer set designed in this study can be a universal rye-specific PCR-based marker and will be utilized in a large-scale screening for wheat plants carrying rye segments that are too small to detect cytologically.

This study was supported by Grant-in-Aid for Scientific Research (A) (No. 123066001) from the Ministry of Education, Science, Sports, and Culture, Japan. Contribution No. 582 from the Laboratory of Plant Genetics, Graduate School of Agricul- 
ture, Kyoto University, Japan.

\section{REFERENCES}

Brunell, M. S., Lukaszewski, A. J., and Whitkus, R. (1999) Development of arm-specific RAPD marker for rye chromosome $2 \mathrm{R}$ in wheat. Crop Sci. 39, 1702-1706.

Endo, T. R., Shi, F., and Tsvetkov, K. S. (1998) Genetic Induction of chromosomal structural changes of alien chromosomes in common wheat. In: Proc. $9_{\text {th }}$ Int. Wheat Genet. Symp., Saskatoon, Vol. 2 (ed.: A. E. Slinkard), pp. 40-43.

Endo, T. R. (2003) Wheat stocks carrying alien chromosomal segments induced by the gametocidal system. In: Proc. $10_{\text {th }}$ Int. Wheat Genet. Symp., Paestum, Vol. 1 (eds.: N. E. Pogna, M. Romano, E. A. Pogna, and G. Galterio), pp. 69-72.

Friebe, B., Hatchett, J. H., Sears, R. G., and Gill, B. S. (1990) Transfer of Hessian fly resistance from "Chaupon" rye to hexaploid wheat via a $2 B S / 2 R L$ wheat-rye chromosome translocation. Theor. Appl. Genet. 79, 385-389.

Friebe, B., Heun, M., Tuleen, N., Zeller, F. J., and Gill, B. S. (1994) Cytogenetically monitored transfer of powdery mildew resistance from rye into wheat. Crop Sci. 34, 621-625.

Gallego, F. J., Lopez-Solanilla, E., Figueiras, A. M., and Benito, C. (1998) Chromosomal location of PCR fragments as a source of DNA markers linked to aluminium tolerance genes in rye. Theor. Appl. Genet. 96, 426-434.

Graybosch, R. A. (2001) Uneasy unions: quality effects of rye chromatin transfers to wheat. J. Cereal Sci. 33, 3-16.

Iqbal, M. J., and Rayburn, A. L. (1995) Identification of the 1RS rye chromosomal segment in wheat by RAPD analysis. Theor. Appl. Genet. 91, 1048-1053.

Jiang, J., Friebe, B., and Gill, B. S. (1994) Recent advances in alien gene transfer in wheat. Euphytica 73, 199-212.

Ko, J.-M., Do. G.-S., Suh, D.-Y., Seo, B.-B., Shin, D.-C., and Moon, H.-P. (2002) Identification and chromosomal organization of two rye-specific RAPD products useful as introgression markers in wheat. Genome 45, 157-164.

Koebner, R. M. D. (1995) Generation of PCR-based markers for the detection of rye chromatin in a wheat background. Theor. Appl. Genet. 90, 740-745.

Lee, J. H., Graybosch, R. A., and Lee, D. J. (1993) Detection of rye chromosome $2 \mathrm{R}$ using the polymerase chain reaction and sequence-specific DNA primers. Genome 37, 19-22.

Lee, J. H., Graybosch, R. A., Kaeppler, S., and Sears, R. G. (1995) Molecular markers for a 2BS/2RL wheat-rye chromosomal translocation. Annu. Wheat Newslett. 41, 281.

Lukaszewski, A. J. (2000) Manipulation of the 1RS.1BL translo- cation in wheat by induced homoeologous recombination. Crop Sci. 40, 216-225.

Lukaszewski, A. J., Rybka, K., Korzun, V., Malyshev, S. V., Lapinski, B., and Whitkus, R. (2004) Genetic and physical mapping of homoeologous recombination points involving wheat chromosome 2B and rye chromosome 2R. Genome 47, 3645.

Ma, X.-F., Wanous, M. K., Houchins, K., Rodriguez, Milla, M. A., Goicoehea, P. G., Wang, Z., Xie, M., and Gustafson, J. P. (2001) Molecular linkage mapping in rye (Secale cereale L.). Theor. Appl. Genet. 102, 517-523.

Masoudi-Nejad, A., Nasuda, S., McIntosh, R. A., and Endo, T. R. (2002) Transfer of rye chromosome segments to wheat by a gametocidal system. Chromosome Res. 10, 349-357.

McIntyre, C. L., Pereira, S., Moran, L. B., and Appeles, R. (1990) New Secale cereale (rye) DNA derivatives for the detection of rye chromosome segment in wheat. Genome 33, 635640.

Murata, M., Nakata, T., and Yasumuro, Y. (1992) Origin and molecular structure of a midget chromosome in a common wheat carrying rye cytoplasm. Chromosoma 102, 27-31.

Philipp, U., Wehling, and P., Wricke, G. (1994) A linkage map of rye. Theor. Appl. Genet. 88, 243-248.

Ribeiro-Carvalho, C., Guedes-Pinto, H., Heslop-Harrison, J. S., and Schwarzacher, T. (2001) Introgression of rye chromatin on chromosome 2D in the Portuguese wheat landrace "Barbela". Genome 44, 1122-1128.

Sandery, M. J., Forster, J. W., Blunden, R., and Jones, R. N. (1990) Identification of a family of repeated sequences on the rye B chromosome. Genome 33, 908-913.

Schlegel, R., and Korzun, V. (1997) About the origin of 1RS.1BL wheat-rye chromosome translocations from Germany. Plant Breeding 116, 537-540.

Singh, N. K., Shepherd, K. W., and McIntosh, R. A. (1990) Linkage mapping of genes for resistance to leaf, stem and stripe rusts and $\omega$-secalins on the short arm of rye chromosome 1R. Theor. Appl. Genet. 80, 609-616.

Tomita, M., Shinohara, K., and Morimoto, M. (2003) Amplification and elimination of a new class of transposon-like element through the evolution of Triticeae genome. In: Proc. $10_{\text {th }}$ Int. Wheat Genet. Symp., Paestum, Vol. 1 (eds.: N. E. Pogna, M. Romano, E. A. Pogna, and G. Galterio), pp. 4650.

Tomita, M., and Yoshikawa, M. (2003) A new class of transposon-like gene Revolver is valuable for molecular breeding in wheat. In: Proc. and abstracts of the International Colloquium on Plant Biotechnology, Sakai, p. 187. 